\title{
Métodos de triagem nutricional em pacientes ginecológicas hospitalizadas
}

\author{
Nutritional screening methods in hospitalized gynecological patients
}

DOI: 10.37111/braspenj.2020354004

Amanda da Silva Paiva'

Larissa Gens Guilherme²

Maria Goretti Pessoa de Araúio Burgos ${ }^{3}$

\section{Unitermos:}

Triagem. Estado nutricional. Ginecologia. Desnutrição. Avaliação nutricional.

\section{Keywords:}

Triage. Nutritional status. Gynecology. Malnutrition. Nutritional assessment.

\section{Endereço para correspondência:}

Maria Goretti Pessoa de Araújo Burgos.

Rua Baltazar Pereira 70 - apto 601 - Boa

Viagem - Recife, PE, Brasil - CEP: 51011-550.

E-mail: gburgos@hotlink.com.br

\section{Submissão:}

19 de abril de 2020

\section{Aceito para publicação}

8 de dezembro de 2020

\section{RESUMO}

Introdução: A desnutrição hospitalar caracteriza-se como um expressivo problema de saúde pública, por isso, a triagem nutricional se destaca como uma ferramenta prévia para identificar o indivíduo em risco nutricional ou desnutrido, possibilitando uma intervenção mais adequada. Método: Estudo descritivo transversal, onde foram utilizados três instrumentos de triagem nutricional: Avaliação Subjetiva Global (ASG), Nutritional Risk Screening -2002 (NRS-2002), Malnutrition Universal Screening Tool (MUST), aplicados nas primeiras 48 horas de admissão hospitalar, pelo mesmo pesquisador. Resultados: Foram avaliadas 150 mulheres, com idade de 42,69 $\pm 11,84$ anos (20 - 83 anos), com diagnóstico principal de miomatose (70\%) nas adultas e, nas idosas, de neoplasias ginecológicas (50\%). A maioria das adultas não apresentava comorbidades associadas (80\%), enquanto a hipertensão arterial foi a mais prevalente nas idosas (30\%). O MUST detectou $41,3 \%$ de risco nutricional, enquanto o NRS-2002 apenas $21,3 \%$. Comparando o MUST e NRS-2002 com a ASG como padrão ouro, a maior sensibilidade ocorreu com o MUST (42,4\%), Kappa 3,4\%. Conclusão: O MUST evidenciou maior sensibilidade para detectar risco nutricional nesta população, e teve significativamente menor tempo de aplicação.

\section{ABSTRACT}

Introduction: Hospital malnutrition is characterized as a significant public health problem, so nutrition screening stands out as a prior tool to identify the individual at nutritional risk or malnourished, enabling a more appropriate intervention. Methods: Cross-sectional descriptive study, where three nutritional screening tools were used: ASG, Nutritional Risk Screening-2002 (NRS-2002), Malnutrition Universal Screening Tool (MUST), applied in the first 48 hours of hospital admission by the same researcher. Results: 150 women aged 42,69 $\pm 11,84$ years (20 - 83 years) were evaluated, in adults the major diagnosis was myomatosis $(70 \%)$ and elderly, gynecological malignancies (50\%). Most adults did not have associated comorbidities $(80 \%)$, while arterial hypertension was the most prevalent in the elderly (30\%). MUST detected $41.3 \%$ nutritional risk, while NRS-2002 only 21.3\%. Comparing the MUST and NRS-2002 with the ASG as gold standard, the greatest sensitivity occurred with the MUST (42.4\%), Kappa 3,4\%. Conclusion: MUST showed greater sensitivity to detect nutritional risk in this population and had significantly shorter application time.

1. Especialista em Nutrição e Saúde da Mulher, Hospital das Clínicas, Universidade Federal de Pernambuco, Recife, PE, Brasil.

2. Graduada em Nutrição, Departamento de Nutrição, Universidade Federal de Pernambuco, Recife, PE, Brasil.

3. Pós-Doutora em Nutrição Clínica, Departamento de Nutrição, Universidade Federal de Pernambuco, Recife, PE, Brasil. 


\section{INTRODUÇÃO}

A desnutrição hospitalar caracteriza-se como um expressivo problema de saúde pública, estando associada à elevação de morbidade, à suscetibilidade a infecções e ao aumento da mortalidade de indivíduos hospitalizados ${ }^{1,2}$.

De acordo com revisão sistemática que avaliou 66 estudos, incluindo 29.474 pacientes de 12 países da América Latina, cerca de 40-60\% eram considerados desnutridos no momento da admissão e esse percentual aumentava à medida que o período de permanência se prolongava ${ }^{3}$. Os dados obtidos pelo Inquérito Brasileiro de Avaliação Nutricional Hospitalar (IBRANUTRI) revelou que $48,1 \%$ dos 4 mil pacientes hospitalizados na rede pública do Brasil apresentavam algum grau de desnutrição, sendo que $31,8 \%$ destes já se encontravam desnutridos nas primeiras 48 horas de admissão ${ }^{4}$.

Estudos relatam a importância do cuidado nutricional no momento da admissão hospitalar, o que pode contribuir para melhorar e/ou manter o bom estado nutricional, evitando ou minimizando complicações. A identificação precoce do risco nutricional (RN) ou desnutrição possibilita uma intervenção de cuidados mais adequados ${ }^{5-7}$.

O RN é definido pela presença de fatores que aumentam a probabilidade de acarretar a deterioração do estado nutricional, sendo importante não só identificar a desnutrição, como também o risco de ela vir a acontecer ${ }^{6}$. Neste sentido, a triagem nutricional se destaca como uma ferramenta prévia para identificar o indivíduo em RN ou desnutrido, necessitando posteriormente de uma avaliação nutricional mais específica ou complementar ${ }^{5,6}$.

Atualmente, existem diversas ferramentas de triagem nutricional validadas e disponíveis na literatura. Dentre elas, destacam-se a Avaliação Subjetiva Global (ASG), - Instrumento de Triagem Universal para Desnutrição (MUST - Malnutrition Universal Screening Tool) e a Triagem de Risco Nutricional (NRS-2002 - Nutritional Risk Screening), tendo cada uma suas particularidades. Entretanto, no Brasil, ainda não há um consenso quanto ao melhor método a ser utilizado'.

A ASG é um método considerado padrão ouro, desenvolvida por Baker et al. ${ }^{8}$ e padronizada por Detsky et al. ${ }^{9}$. Este método inicialmente era utilizado exclusivamente para pacientes cirúrgicos e, posteriormente, passou a ser utilizado nas demais especialidades clínicas. O MUST foi desenvolvido por um grupo multidisciplinar britânico Malnutrition Advisory Group (MAG), um comitê permanente da British Association for Parenteral and Enteral Nutrition e apoiado por órgãos envolvidos em nutrição humana $^{10}$. Este método é aplicado em adultos e idosos, em ambulatórios ou hospitais. A The European Society for Clinical Nutrition and Metabolism (ESPEN) criou o NRS-2002, que pode ser utilizado em adultos ou idosos, no contexto hospitalar ${ }^{11}$.

Hertlein et al. ${ }^{12}$ foram os primeiros pesquisadores a adaptarem NRS 2002 para avaliar a desnutrição em pacientes de ginecologia, eles realizaram uma mínima alteração da NRS relativo à categorização dos diagnósticos das doenças ginecológicas, sendo a gravidade da doença um dos componentes desta ferramenta ${ }^{12}$. Atualmente, não existe uma ferramenta específica validada para pacientes ginecológicas hospitalizadas, como também existem poucos dados sobre a prevalência da desnutrição nesta população.

De acordo com Soares ${ }^{13}$, a eficiência do método da triagem nutricional pode ser analisada por meio de duas medidas denominadas sensibilidade e especificidade. A sensibilidade mede a capacidade do exame de identificar a desnutrição. Por outro lado, a especificidade mensura a capacidade do exame de excluir de forma correta os indivíduos que não apresentam a desnutrição ${ }^{13}$.

Contudo, torna-se essencial a escolha de um método mais sensível, simples e de fácil aplicação, que permita aos profissionais identificar os indivíduos em RN ou desnutrição, adequando-o com as especificidades da população atendida.

\section{MÉTODO}

Estudo descritivo transversal, de natureza quantitativa, aprovado pelo Comitê de Ética em Pesquisa do Centro de Ciência da Saúde Universidade Federal de Pernambuco (UFPE), sob o CAAE: 51415815.9.0000.5208, desenvolvido na enfermaria de ginecologia, de um hospital universitário de Recife, PE.

A coleta foi realizada com até, no máximo, 48 horas do início do internamento, no período de março a agosto de 2016 . A captação das mulheres foi feita a partir da assinatura do Termo de Consentimento Livre e Esclarecido (TCLE), após serem detalhados os objetivos do estudo. Dados referentes à idade e ao motivo da internação foram coletados no prontuário, posteriormente sendo aplicados os três instrumentos de triagem: MUST, NRS-2002 e ASG.

$\mathrm{Na}$ padronização dos resultados da triagem nutricional para análise estatística, foi utilizada a classificação adaptada por Bezerra et al. ${ }^{14}$. No MUST, foi usada a classificação: sem risco (baixo risco, de acordo com a classificação original) e com RN (médio e alto risco da classificação original), enquanto para NRS-2002 mantevese a classificação legítima. Na ASG, as pacientes foram 
categorizadas como aquelas com RN: desnutrida leve, moderada ou grave na classificação original; e sem risco, aquelas bem nutridas na classificação original.

$\mathrm{Na}$ análise estatística, os dados foram expressos por meio de frequências absolutas e percentuais para as variáveis categóricas e média, desvio padrão e mediana, para as variáveis numéricas. Para avaliar diferenças significativas entre os percentuais das categorias foi utilizado o teste Qui-quadrado para uma amostra; para avaliar associação entre variáveis categóricas foi utilizado o teste Exato de Fisher e, para a comparação entre as categorias do risco nutricional em relação às variáveis numéricas foi utilizado o teste de Kruskal-Wallis para três categorias ou o teste t-Student com variâncias iguais, para a comparação de duas categorias. No estudo de diagnóstico do risco dos instrumentos NRS-2002 e MUST, em relação ao considerado padrão ouro ASG, foram calculadas as medidas: sensibilidade, especificidade, valor preditivo positivo (VPP), valor preditivo negativo (VNP) e acurácia. Para avaliar o grau de concordância foi obtido o valor de Kappa. A utilização do teste Exato de Fisher ocorreu porque não foi verificada a condição para utilização do teste Qui-quadrado de Pearson. O teste de Kruskal-Wallis foi usado devido à falta de normalidade dos dados e, o teste t-Student, quando a distribuição dos dados se mostrou normal. A verificação da hipótese de normalidade foi realizada por meio do teste de Shapiro-Wilk e, da igualdade de variâncias através do teste $F$ de Levene. A margem de erro utilizada nas decisões dos testes estatísticos foi de 5\%. Os dados foram digitados na planilha EXCEL e o programa utilizado para obtenção dos cálculos estatísticos foram o SPSS (Statistical Package for the Social Sciences) na versão 23.

\section{RESULTADOS}

Foram avaliadas 150 mulheres, predominantemente adultas (93,3\%), com idade média de 42,69 $\pm 11,84$ anos e, as idosas com faixa etária até 83 anos. O IMC foi de $25,89 \pm 5,84 \mathrm{~kg} / \mathrm{m}^{2}$, com classificação distinta pela NRS 2002 e o MUST.

O principal motivo de internação nas adultas foi a miomatose (70\%) e, nas idosas, as neoplasias (50\%). $\mathrm{Na}$ avaliação estatística, foi detectada diferença entre o grupo de patologias do internamento e as comorbidades encontradas, nas duas faixas etárias (Tabela 1).

$\mathrm{Na}$ triagem nutricional, realizada pelas três ferramentas (Tabela 2), identificou-se maior frequência de risco pela ASG, independente da faixa etária. Do mesmo modo, foi o instrumento de triagem com maior tempo de aplicação, variando em torno de 2 minutos.
Tabela 1 - Avaliação das patologias e comorbidades, segundo a idade de pacientes do Hospital Universitário da UFPE, 2016.

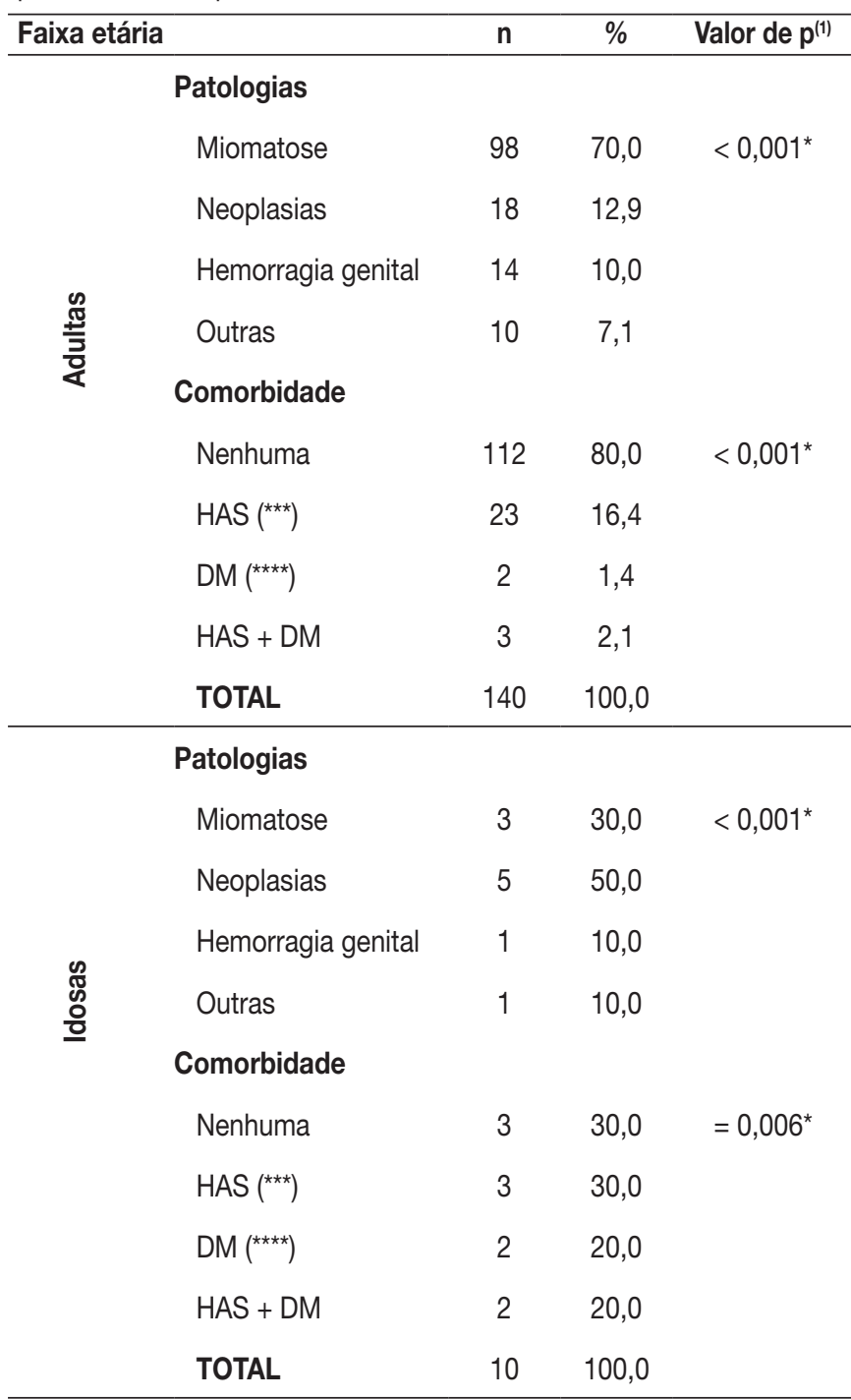

(*) Diferença significativa ao nível de 5,0\%. $\left.{ }^{* *}\right)$ Outras doenças: Doença Inflamatória Pélvica; Síndrome de Fournier; Bartholinite; Teratoma; Cistocele e Retocele; Abscesso ovariano; Cisto simples de ovário. (1): Através do teste Qui-quadrado para uma amostra.

Em relação à comparação entre faixas etárias e instrumentos de risco nutricional, foi observado que as idosas apresentaram maior risco pela NRS-2002 e ASG, enquanto as adultas, maior risco pelo MUST (Tabela 3), sem, no entanto, revelar diferença estatística $(p>0,05)$ entre os dois grupos etários.

Na Figura 1, está demonstrada a prevalência de risco de desnutrição, de acordo com os três métodos, onde a ASG revelou maior risco e a NRS 2002, menor risco.

Na Tabela 4, observa-se que o MUST apresentov elevada sensibilidade $(42,4 \%)$ e especificidade (83,3\%), quando comparado ao ASG como padrão ouro. Ao mesmo tempo, detectou-se baixa sensibilidade na NRS-2002 (22,2\%) e elevada especificidade, porém com reduzido quantitativo. 
Tabela 2 - Distribuição dos pacientes quanto ao risco nutricional diferentes instrumentos de triagem, e seu respectivo tempo de aplicação no Hospital Universitário da UFPE, 2016.

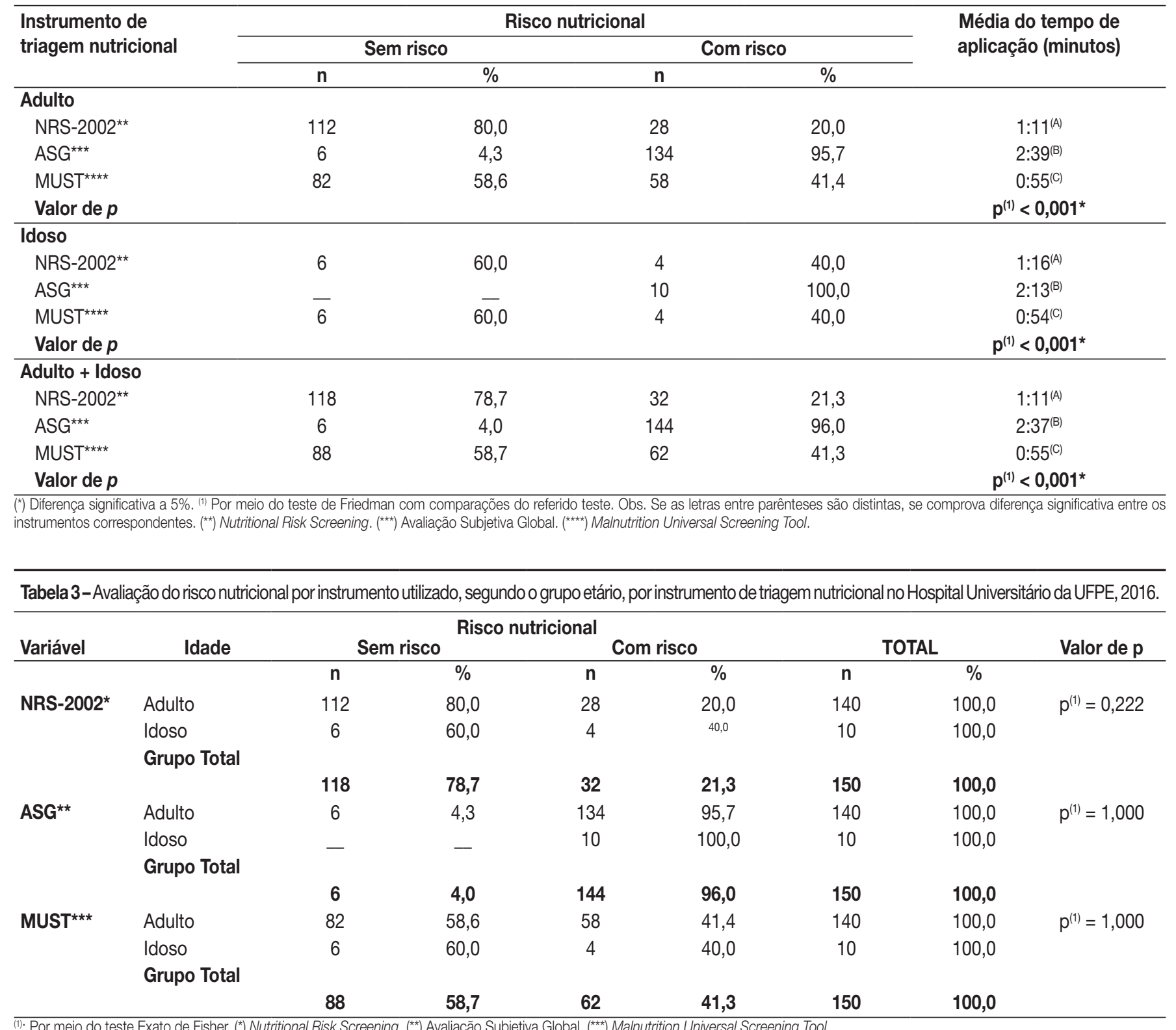

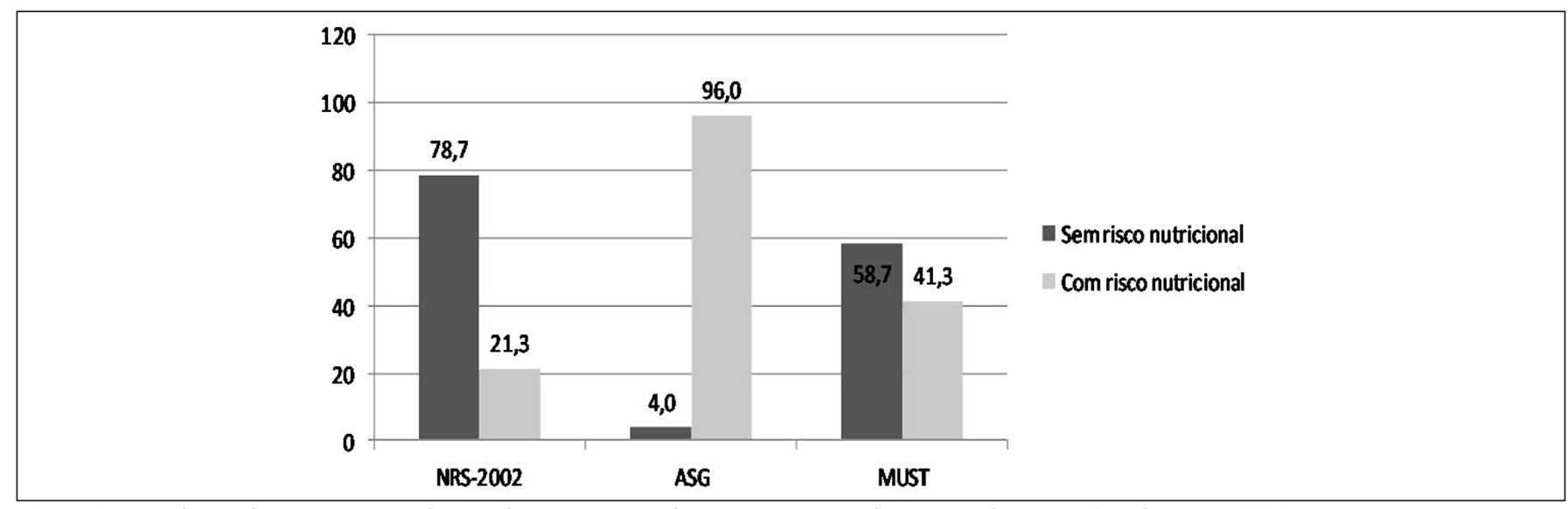

Figura 1 - Prevalência do risco nutricional, segundo o instrumento de triagem nutricional no Hospital Universitário da UFPE, 2016. NRS-2002 = Nutritional Risk Screening - 2002; ASG = Avaliação Subjetiva Global; MUST = Malnutrition Universal Screening Tool. 
Tabela 4 - Avaliação das medidas (percentuais) sensibilidade, especificidade, dos instrumentos NRS-2002 e MUST em relação ao padrão ouro (ASG) no Hospital Universitário da UFPE, 2016.

\begin{tabular}{lcccccc}
\hline Instrumento & $\begin{array}{c}\text { Sensibilidade } \\
(\mathbf{n} 1 / \mathbf{n})\end{array}$ & $\begin{array}{c}\text { Especificidade } \\
(\mathbf{n} 1 / \mathbf{n})\end{array}$ & $\begin{array}{c}\mathbf{V P P}^{*} \\
(\mathbf{n} 1 / \mathbf{n})\end{array}$ & $\begin{array}{c}\text { VPN } \\
(\mathbf{n} 1 / \mathbf{n})\end{array}$ & $\begin{array}{c}\text { Acurácia } \\
(\mathbf{n} 1 / \mathbf{n})\end{array}$ & Kappa \\
\hline NRS-2002 $^{* \star *}$ & $22,2(32 / 144)$ & $100,0(6 / 6)$ & $100,0(32 / 32)$ & $5,1(6 / 118)$ & $25,3(38 / 150)$ & 2,2 \\
MUST $^{\star \star \star \star}$ & $42,4(61 / 144)$ & $83,3(5 / 6)$ & $98,4(61 / 62)$ & $5,7(5 / 88)$ & $44,0(66 / 150)$ & 3,4 \\
\hline
\end{tabular}

*Valor preditivo positivo. $\left.{ }^{(*)}\right)$ Valor preditivo negativo. $\left.{ }^{(* \star}\right)$ Nutritional Risk Screening. ${ }^{(* \star *)}$ Malnutrition Universal Screening Tool.

\section{DISCUSSÃO}

Neste estudo, os resultados oriundos da ASG em adultas e idosas detectaram percentuais semelhantes de RN. Por carência de estudos utilizando estas ferramentas exclusivamente em patologias ginecológicas, a discussão foi realizada com populações mistas.

Bezerra et al. ${ }^{14}$, comparando diferentes instrumentos de triagem nutricional em população de hospital geral, com pacientes predominantemente mulheres adultas, observou que o MUST, independente da faixa etária, conseguiu detectar melhor o RN. Quando separados por grupos, constataram $19,6 \%$ de RN nos adultos e $34,6 \%$ nos idosos ${ }^{14}$, valores inferiores ao deste estudo $(41,4 \%$ e $40 \%$, respectivamente).

Estudo realizado no Brasil em um hospital público, com semelhantes características demográficas (mulheres adultas), reportou RN de 35,3\% e 28,5\% segundo o MUST e NRS-2002, respectivamente, valores próximos ao encontrado neste estudo. Por outro lado, a ASG detectou RN em 32,9\%, resultado oposto a esta pesquisa, fato explicado provavelmente por se tratar exclusivamente de mulheres, portadoras na sua maioria de miomatose e neoplasias, doenças com alto risco de desnutrição'.

Sanches et al. ${ }^{15}$, analisando 111 pacientes clínicos e cirúrgicos, encontraram RN de 73\% e 70,3\%, segundo NRS-2002 e MUST, respectivamente, valores muito elevados em relação aos nossos achados, o que pode ter sido devido ao tempo elevado para aplicação da triagem, pois havia pacientes que foram admitidos em 72 horas sendo triados.

Koren-Hakin et al. ${ }^{16}$, utilizando o MUST e o NRS-2002 em clínica geriátrica ortopédica, identificaram o RN pelo MUST de $20 \%$ ( $14,4 \%$ de RN médio e $6 \%$ de alto risco), valor inferior ao encontrado neste estudo, com $40 \%$ de RN. Ao mesmo tempo em que o NRS-2002 revelou percentual de 37,6\% (32,5\% RN médio e 5,1\% alto risco), índices semelhantes aos nossos achados $(40 \%)^{16}$.

Hertlein et al. ${ }^{12}$, utilizando NRS-2002 em ginecologia, com idade $>18$ anos, internadas em hospital na Alemanha, detectaram 35,8\% de RN, valores superiores ao identificado nesse grupo, fato provavelmente explicado pelas alterações realizadas no método relativas à categorização dos diagnósticos, que pontua a gravidade da doença ${ }^{12}$.
O tempo de aplicação do método é fator importante na escolha da ferramenta de triagem, visto que a rotina do ambiente hospitalar é intensa e, ao mesmo tempo, atendemos pacientes com baixo nível de escolaridade. Um método que requer mais tempo poderá acarretar enfado e desconforto aos pacientes/familiares, podendo provocar viés nas respostas ${ }^{14}$.

Em relação ao tempo médio de aplicação das ferramentas, nota-se divergência com Stratton et al. ${ }^{17}$ em relação MUST, onde encontraram uma média de 3-5 minutos e semeIhança com Bezerra et al. ${ }^{14}$, com achados de 1,1 minutos. Do mesmo modo, na aplicação do NRS-2002, Li et al. ${ }^{18}$ encontraram uma média 7,3 minutos, enquanto Bezerra et al. ${ }^{14}$ referiram 1,4 minutos, valor próximo ao encontrado $(1,11$ minutos). Por falta de resultados na literatura referindo tempo de aplicação com a ASG, ficamos impossibilitados de comparar os nossos resultados com outros autores. Constatamos nesta pesquisa que o tempo de aplicação do MUST por ter sido menor pelo fato de ser o último instrumento a ser aplicado, além de algumas respostas já terem sido respondidas nos questionários anteriores.

Dados de Soares ${ }^{13}$, no Distrito Federal, em clínica cirúrgica, sendo a maioria do sexo feminino e com predomínio de adultos, relata adequada especificidade e reduzida sensibilidade do NRS-2002, concordando com este estudo, no entanto, vale ressaltar o reduzido $n$, para esta análise estática. A especificidade do NRS-2002 pode ser devido ao fato de verificar a gravidade da doença, visto que o MUST caracteriza automaticamente pacientes com doenças graves como de alto risco.

Revisão que investigou pesquisas de validação de ferramentas de triagem nutricional, com ênfase nos valores de sensibilidade e especificidade, encontrou 20 artigos sobre o tema, sendo 12 estudos de validação selecionada. Os autores concluíram que as diferentes metodologias empregadas com diferentes padrões de referências impossibilitam uma análise adequada de valores de sensibilidade e especificidade para essas ferramentas ${ }^{19}$.

Eleger um instrumento de triagem nutricional para ser adotado na rotina do serviço de nutrição, em clínica ginecológica, requer atenção especial em relação aos instrumentos disponíveis e já validados, na facilidade e menor tempo de aplicação. 


\section{CONCLUSÃO}

Os resultados demonstram que o NRS-2002 e o MUST, quando comparados à ASG, evidenciaram maior sensibilidade do MUST e maior especificidade do NRS-2002, na detecção do RN de mulheres adultas e idosas. Além disso, - MUST mostrou-se mais prático e apresentou menor tempo de aplicação, em ambas as faixas etárias. Dessa forma, sugere-se o MUST para triagem nutricional de pacientes ginecológicas hospitalizadas.

\section{AGRADECIMENTOS}

À Secretaria Estadual de Saúde de Pernambuco e ao Programa de Residência em Nutrição do Hospital das Clínicas da UFPE.

\section{REFERÊNCIAS}

1. Raupp D, Silva FM, MarcadentiA, Rabito EI, Silva Fink J, Becher $\mathrm{P}$, et al. Nutrition screening in public hospital emergency rooms: Malnutrition Universal Screening Tool and Nutritional Risk Screening-2002 can be applied. Public Health. 2018;165:6-8.

2. Leandro-Merhi VA, Costa CL, Saragiotto L, Aquino JLB. Nutritional indicators of malnutrition in hospitalized patients. Arq Gastroenterol. 2019;56(4):447-50.

3. Correia MITD, Perman MI, Waitzberg DL. Hospital malnutrition in Latin America: a systematic review. Clin Nutr. 2017;36(4):958-67.

4. Waitzberg DL, Caiaffa WT, Correia MI. Hospital malnutrition: the Brazilian national survey (IBRANUTRI): a study of 4000 patients. Nutrition. 2001;17(7-8):573-80.

5. BarbosaAAO, VicentiniAP, LangaFR. Comparison of NRS-2002 criteria with nutritional risk in hospitalized patients. Cien Saude Colet. 2019;24(9):3325-34.

6. Lima GES, Silva BYC. Ferramentas de triagem nutricional: um estudo comparativo. BRASPEN J. 2017;32(1):20-4.

7. Martín Palmero Á, Serrano Pérez A, Chinchetru Ranedo MJ, Cámara Balda A, Martínez de Salinas Santamari MÁ, Villar García $G$, et al. Malnutrition in hospitalized patients: results from La Rioja. Nutr Hosp. 2017;34(2):402-6.
8. Baker JP, Detsky AS, Wesson DE, Wolman SL, Stewart S, Whitewell J, et al. Nutritional assessment: a comparison of clinical judgment and objective measurements. N Engl J Med. 1982;306(16):969-72.

9. Detsky AS, McLaughlin JR, Baker JP, Johnston N, Whittaker $\mathrm{S}$, Mendelson RA, et al. What is subjective global assessment of nutritional status? JPEN J Parenter Enteral Nutr. 1987;11(1):8-13.

10. British Association for Enteral and Parenteral Nutrition. The MUST Explanatory Booklet. A guide to malnutrition universal screening tool (MUST) for adults. Malnutrition Advisory Group (MAG). [Acesso em 11 de março de 2020]. Disponível em: https://www.bapen.org.uk/screening-and-must/must/ must-toolkit/the-must-explanatory-booklet

11. Kondrup J, Allison SP, Elia M, Vellas B, Plauth M. ESPEN guidelines for nutrition screening 2002. Clin Nutr. 2003;22(4):415-21.

12. Hertlein L, Kirschenhofer A, Fürst S, Beer D, GößC, Lenhard M, et al. Malnutrition and clinical outcome in gynecologic patients. Eur J Obstet Gynecol Reprod Biol. 2014;174(1):137-40.

13. Soares GO. Comparação da especificidade e da sensibilidade entre os métodos de triagem nutricional NRS 2002 e MST em pacientes cirúrgicos de Santa Maria, Distrito Federal. Rev Nutr Vigilân Saúde. 2016;3(1):26-31.

14. Bezerra JD, Dantas MAM, Vale SHL, Dantas MMG, Leite LD. Aplicação de instrumentos de triagem nutricional em hospital geral: um estudo comparativo. Rev Ciênc Saúde. 2012;5(1):9-15.

15. Sanches FLFZ, Ferreira T, Guimarães RCA. Risco nutricional em pacientes hospitalizados: comparação de três protocolos de triagem nutricional. Multitemas. 2018;23(55):245-63.

16. Koren-Hakim T, Weiss A, Hershkovitz A, Otzrateni I, Anbar $\mathrm{R}$, Nevo RFG, et al. Comparing the adequacy of the MNA-SF, NRS-2002 and MUST nutritional tools in assessing malnutrition in hip fracture operated elderly patient. Clin Nutr. 2016;35(5):1053-8.

17. Stratton RJ, Hackston A, Longmore D, Dixon R, Price S, Stroud $\mathrm{M}$, et al. Malnutrition in hospital outpatients and inpatients: prevalence, concurrent validity and ease of use of the 'malnutrition universal screening tool' ('MUST') for adults. Br J Nutr. 2004;92(5):799-808.

18. Li XY, Yu K, Yang Y, Wang YF, Li RR, Li CW. Nutritional risk screening and clinical outcome assessment among patients with community-acquired infection: a multicenter study in Beijing teaching hospitals. Nutrition. 2016;32(10):1057-62.

19. Vale FCG, Logrado MHG. Estudos de validação de ferramentas de triagem e avaliação nutricional: uma revisão acerca da sensibilidade e especificidade. Com Ciências Saúde. 2013;22(4):31-46.

Local de realização do estudo: Hospital das Clínicas da Universidade Federal de Pernambuco, Recife, PE, Brasil.

Conflito de interesse: Os autores declaram não haver. 